\section{Thyroid carcinoma presenting as Pancoast's syndrome}

\author{
A Rabano, M La Sala, P Hernandez, \\ $\mathrm{J}$ L Barros
}

\begin{abstract}
An unusual clinical presentation of papillary carcinoma of the thyroid is presented, which adds to the infrequent causes of the thoracic inlet syndrome.
\end{abstract}

The thoracic inlet syndrome, with brachial pain and a classical Claude-Bernard-Horner syndrome, is due in most cases to a primary malignant neoplasm of the pulmonary apex. According to most authors, this so called "Pancoast tumour" can be managed through combination treatment that includes radiation and radical surgery. Since the original description by Pancoast in $1932^{1}$ the list of causes of this syndrome has increased. Fine needle aspiration biopsy has been suggested as an adequate way of obtaining diagnostic material. $^{2}$

We present a case of primary carcinoma of the thyroid presenting as Pancoast's syndrome, in which fine needle aspiration biopsy yielded an erroneous diagnosis of right superior pulmonary sulcus tumour.

\section{Case report}

A 45 year old man presented in June 1985 with a two month history of pain in his right arm that radiated to the right hemithorax and neck, ipsilateral palpebral ptosis, and enophthalmos. He had been a heavy smoker for

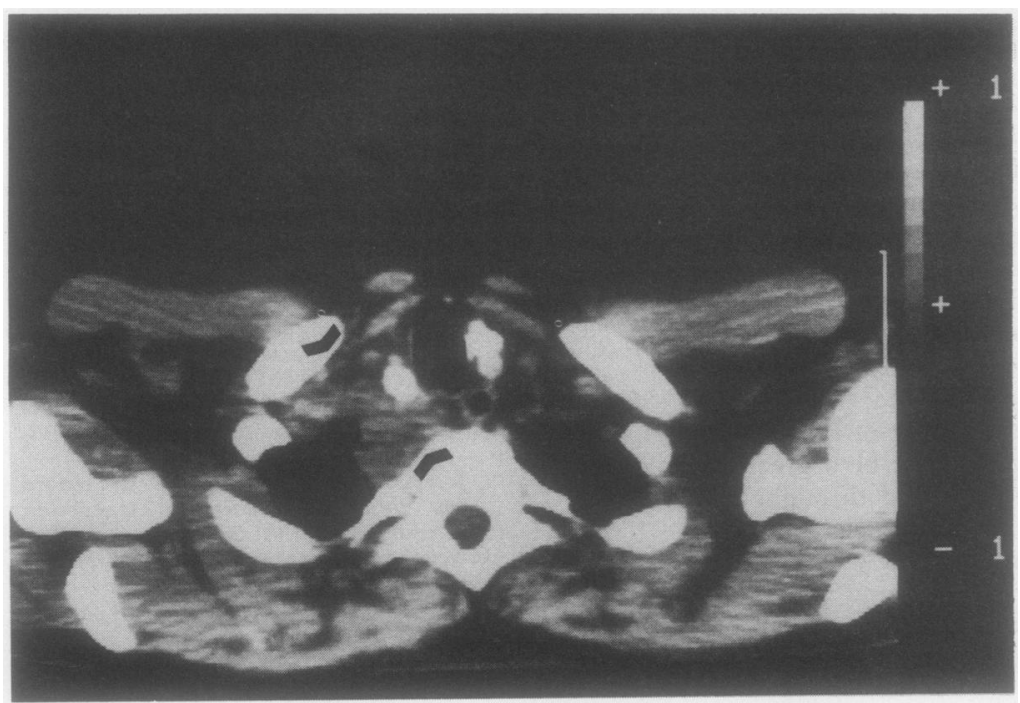

Figure 1 Computed tomography section of the upper thorax showing (arrowheads) a right paratracheal and retrotracheal extension of the tumour apparently affecting the right pulmonary sulcus. Note the presence of bilateral paratracheal calcific nodes primarily interpreted as old tuberculous lesions, though corresponding to metastases of a thyroid carcinoma. many years. Physical examination disclosed a fully developed right Horner's syndrome without sensory or motor impairment of the right upper limb. On examination of the neck small bilateral enlarged cervical lymph nodes were observed. Examination of the thyroid showed nothing abnormal. Computed tomography (fig 1) showed a soft tissue mass affecting the right pulmonary apex in the paravertebral region, extending through the retrotracheal space at that level and displacing the oesophagus to the left side. A Pancoast tumour was suspected. Multiple lateral cervical, paratracheal, and mediastinal bilateral calcified lesions were interpreted as old tuberculous lesions. A fine needle aspiration biopsy of the mass was performed under computed tomographic control, and the appearances were reported to be consistent with an undifferentiated carcinoma. A diagnosis of right superior pulmonary sulcus tumour was made. Combination treatment was planned, and radiotherapy was started with a total dose of $60 \mathrm{~Gy}$.

In October 1985 the patient was admitted for surgery. He was clinically euthyroid, and physical examination showed the neck to be unchanged. At that time computed tomography showed a significant reduction of the apical lung mass with a persistent soft tissue image. Mediastinoscopy showed numerous pretracheal and paratracheal lymph nodes, which on frozen section biopsy contained metastatic deposits of thyroid carcinoma with a predominantly follicular pattern and occasional papillary areas. During the procedure the apex of the right lung was explored and found to be normal. Surgery was subsequently accomplished through a transverse neck incision and a separate longitudinal sternotomy. Total thyroidectomy with bilateral cervical and mediastinal lymph node dissection and thymectomy were performed. The right pulmonary apex was found to be normal. On examination of the surgical specimen both thyroid lobes measured $7 \times 4 \mathrm{~cm}$. The right lobe contained two whitish hard, partly calcified, irregular nodules of 2.0 and $1.0 \mathrm{~cm}$ diameter showing extrathyroid extension. The left lobe contained two smaller nodules limited to the thyroid gland Histologically, all the nodules were carcinomatous and there were tumour deposits in the cervical and upper mediastinal lymph nodes. The tumour in the thyroid was predominantly follicular but in the lymph node metastases there was a conspicuous papillary growth pattern. The neoplastic cells showed ground glass nuclei and nuclear grooving (fig 2), indicating a diagnosis of papillary carcinoma of the thyroid with a large follicular component. ${ }^{3}$ In addition, occasional less differentiated areas were found in the tumour metastases.

The patient was operated on two months later because of the presence of one rapidly growing left cervical lymph node metastasis, and he lived with no evidence of disease until June 1988, when he died of an acute myocardial infarction. Necropsy was not performed. 
Figure 2 Trabecular and follicular growth area in the primary thyroid tumour. The presence of ground glass nuclei and nuclear grooving in most of the cells allows the diagnosis of papillary carcinoma of the thyroid to be made.

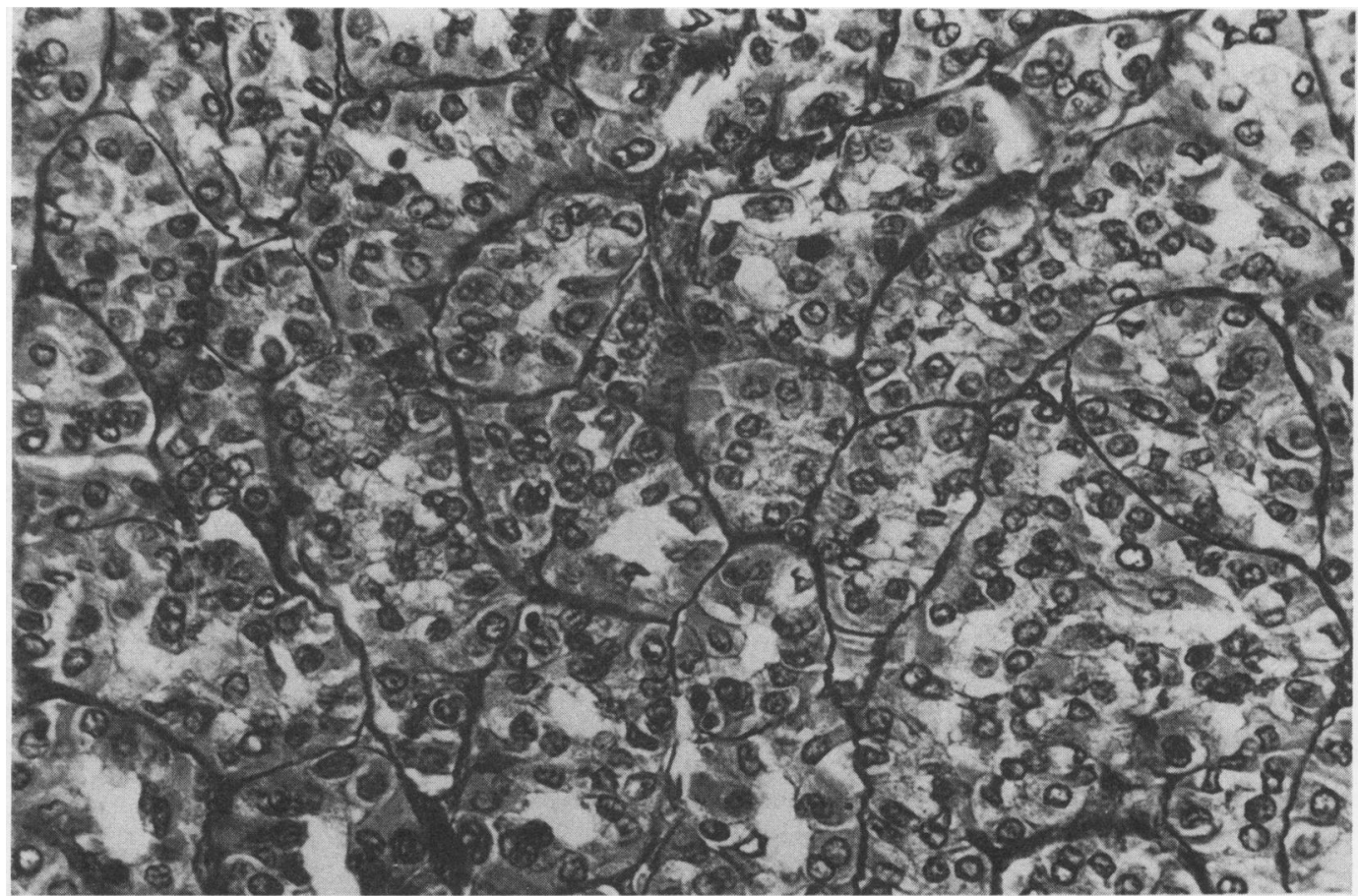

\section{Discussion}

A superior sulcus tumour can be diagnosed in $95 \%$ of cases on a clinical and radiological basis. Although still a much debated issue, combined surgery and radiotherapy appears to be the optimal treatment in these patients, provided that mediastinal structures are not diseased. A $10 \%$ five year survival rate may be achieved with radiotherapy alone; survival is slightly better with surgery only, and a significantly longer survival is attained after combined treatment. ${ }^{4}$

Nevertheless, any tumoral or non-tumoral process affecting the superior pulmonary sulcus may result in Pancoast's syndrome..$^{5-10}$ As most such lesions will produce radiological images consistent with a primary apical lung tumour, it is important that an accurate pathological diagnosis is made. Supraclavicular thoracotomy and fine needle aspiration biopsy have been advocated for this. As in our case, however, a malignant neoplasm displaying undifferentiated areas may give rise to diagnostic errors that may delay proper treatment for these patients. The only two reported cases of Pancoast's syndrome due to primary thyroid carcinoma resulted from the extrathyroid extension of an undifferentiated carcinoma. ${ }^{5}$ Pathological examination of the surgical specimen in the present case yielded a diagnosis of papillary carcinoma of the thyroid with a large follicular component, though the tumour showed occasional less differentiated areas. In the present case mediastinoscopy with frozen section examination gave an accurate diagnosis, which resulted in appropriate treatment.

1 Pancoast HK. Superior pulmonary sulcus tumor: tumor characterized by pain, Horner's syndrome, destruction of bone and atrophy of hand muscles. JAMA 1932;99: 1391-2.

2 Shaw RR. Pancoast's tumour. Ann Thorac Surg 1984;37:343-5.

3 Hedinger CHR, Williams ED, Sobin LH. Histological typing of thyroid tumours. In: World Health Organisation. International histological classification of tumours. 2nd ed. Berlin: Springer, 1988.

4 Martini N, Bains MS, McCormack P, Kaiser LR, Burt ME, Pomerantz AH. Surgical treatment in non-small cell carcinoma of the lung: the memorial Sloan-Kettering experience. In: Hoogstraten B, Addis BJ, Hansen HH, Martini N, Spiro SG, eds. Lung tumours. Current treatment of cancer. UICC. Berlin: Springer, 1988.

5 Herbut PA, Watson JS. Tumor of the thoracic inlet producing the Pancoast syndrome: a report of 17 cases and a review of the literature. Arch Pathol 1946;42:88-103.

6 Rockoff SD. Apical lung cancer masquerading as a Pancoast tumor. $A J R$ 1982;139:163-5.

7 Stathatos C, Kontaxis AN, Zafiracopoulos P. Pancoast's syndrome due to hydatid cysts of the thoracic outlet. $J$ Thorac Cardiovasc Surg 1969;58:764-8.

8 Chocarro A, Labanda F, Martinez E y Pereda JM. Sindrome de Pancoast como primera manifestación de hepatocarcinoma. Medicina Clínica 1986;19:56.

9 Johnson DH, Hainsworth JD, Greco FA. Pancoast's syndrome and small cell lung cancer. Chest 1982;82:602-5.

10 Campbell P, Neil T, Wake PN. Horner's syndrome caused by an intercostal chest drain. Thorax 1989;44:305-6. 